\title{
Sobrevilla, David. Escritos mariateguianos. Artículos y reseñas en torno a José Carlos Mariátegui y su obra. Lima, UIGV, 2012; 244 pp.
}

\author{
Segundo Montoya Huamaní \\ Universidad Nacional Mayor de San Marcos
}

«Escritos mariateguianos» es un libro que reúne un conjunto de ocho artículos y siete reseńas publicados entre 1987 y el 2010, dedicado a la memoria del menor de los hijos del Amauta recientemente fenecido, Javier Mariátegui Chiappe (Perú, 1927-2008), y al peruanista, crítico literario y reconocido estudioso de Mariátegui, Antonio Melis (Italia-1942). Antes de empezar con la reseña debemos definir cartesianamente las «reglas del método» para el abordaje del texto que por su diversidad temática demanda un amplio conocimiento de casi la totalidad de obras escritas por Mariátegui sobre crítica literaria, política nacional y mundial, historia europea, economía colonial peruana, religión, psicoanálisis, filosofía, educación y prensa.

La primera regla consiste en describir fielmente las ideas vertidas por el autor, la segunda consiste en explorar las nuevas propuestas hermenéuticas, es decir, lecturas bajo nuevas claves de interpretación, y la tercera y última consiste en formular observaciones críticas. Para ello seguiremos el mismo orden en el que aparecen los artículos tomando en cuenta las dos reglas esenciales de la interpretación de Sobrevilla, a saber: 1) estudiar a Mariátegui con la misma seriedad y espíritu crítico que a todos los autores occidentales y 2) (tratar de )ser imparcial, es decir, (intentar) prescindir de todo presupuesto ideológico y político.

Asimismo, hay alusiones implícitas a las tesis de Raimundo Prado y a las de Aníbal Quijano que, obviamente, el autor no comparte; también, reproduce sin miramientos la tesis de que es indispensable depurar y renovar íntegramente la teoría del Amauta con el mismo espíritu y afán liquidacionista, mas no «revisionista» de hace siete años, vertidas en su libro «El marxismo de Mariátegui y su aplicación a los 7 ensayos» (2005).

\section{Primer artículo: La visión estética y de la literatura de su tiempo}

En la introducción, Sobrevilla nos recuerda una verdad de Perogrullo o lugar común dentro de la literatura marxista, me refiero al hecho de que en Marx al igual que en Mariátegui no hay un tratado de estética, pero sí hay propuestas 
sobre el arte y la estética, obviamente desarticuladas, sobre todo en los "Los manuscritos económicos filosóficos de 1844» e «Ideología alemana» en el caso de Marx y en «Signos y obras», "emas de educación», "Ideología y política» y otros textos, por supuesto en Mariátegui. Ahora bien, Sobrevilla procede a «reconstruir» las ideas sobre estética en Mariátegui que se encuentran en sus trabajos literarios. Al primer capítulo sobre "La visión estética» corresponden las siguientes ideas: en Mariátegui no hay una definición del arte, pero sí algunas precisiones sobre el fenómeno artístico, a saber, no hay una superioridad de la vida sobre el arte, ni a la inversa; para la captación de lo real es fundamental el papel que juega la imaginación en el arte, de ahí que la fantasía y la ficción estén limitadas por lo real; el arte es verosímil según sus propias leyes por lo menos hasta un cierto punto, y no lo que el consenso público hace pasar por realidad; el arte es un fenómeno fundamentalmente histórico; la literatura como expresión artística forma parte de la superestructura cultural sustentada en la estructura económica.

De lo dicho anteriormente, Sobrevilla enumera los tres criterios de Mariátegui para juzgar el valor de una obra de arte. El primero es que la obra artística cree y capte en todo cuanto sea posible la realidad de la época, es decir, que sea una obra realista; el segundo es la novedad de la obra de arte; el tercero es que la obra de arte obre revolucionariamente, o cuando menos rebelándose contra la propia época. Al segundo capítulo sobre la «visión de la literatura de su tiempo» corresponden las siguientes ideas: si la época es de crisis económica y política, la cultura y el arte en particular no podrían sustraerse a ella. Asimismo, Mariátegui constata que en su época habían envejecido los conceptos y las formas clásicas. Empero la crisis del capitalismo ha provocado no solo la caducidad de las viejas formas artísticas, también ha traído la pérdida de la unidad del arte y la aparición de tendencias artísticas.

Sobre el arte y a la literatura de un país dependiente como el Perú, Mariátegui es bastante claro en cuanto a que como hecho artístico la literatura peruana es de origen español; en rigor, no hubo literatura quechua. Además propone un esquema que divide la literatura peruana en tres periodos: el colonial, el cosmopolita y el nacional. Se trata de un esquema literario y no sociológico.

Observaciones: Sobrevilla señala verdades que se deducen fácilmente de las obras de Mariátegui y que no demandan ningún tipo de análisis profundo y riguroso, como por ejemplo: no hay una definición stricto sensu del arte, la política, la educación, la filosofía, etcétera, o que la literatura, como forma de conciencia social, corresponda a la superestructura. Lo mismo ocurre al momento de enumerar los tres criterios con los que Mariátegui juzga el valor de una obra de arte: 1) realista, 
2) novedoso y 3) revolucionario. Por otro lado, la periodificación de la literatura peruana propuesta por Mariátegui: 1)colonial, 2) cosmopolita y 3)nacional, es tanto sociológica como literaria y no solo literaria, como dice Sobrevilla, repitiendo a Mariátegui porque en el Amauta se opera algo que él llama unimismamiento $^{1}$, es decir, se unifican las concepciones estéticas con las concepciones morales, políticas, religiosas y hasta filosóficas, que sin dejar de ser concepciones estrictamente estéticas no pueden operar independientemente. Pero, ¿̨por qué dice Mariátegui que su criterio es literario? Porque al no utilizar el esquema marxista de literatura feudal, burguesa y proletaria sus planteamientos no son calco y copia sino reformulación critica, y porque él ya se esperaba la avalancha de críticas de los académicos universitarios. Entonces ¿cuál es el mérito de este artículo publicado en 1992 por Sobrevilla? Su mérito reside en que el artículo sirve como un excelente resumen de las tesis de Mariátegui sobre el arte y la literatura.

\section{Segundo artículo: "La visión de la política mundial en La escena contempo- ránea»}

En opinión de Sobrevilla La escena contemporánea tiene dos partes: una visión de la política mundial hacia 1925 y otra del rol revolucionario de los intelectuales. Sobrevilla se ocupará solo de la primera parte. En este texto ofrece una exposición de los resultados a los que Mariátegui había llegado en la historia de la crisis mundial y luego una presentación de la visión mariateguiana de la política mundial en la escena contemporánea. A decir de Sobrevilla, Mariátegui tomó su idea de la crisis mundial de la lectura del libro de Oswaldo Spengler, La decadencia de Occidente. El Amauta propone una interpretación marxista de la tesis spengleriana sobre la decadencia de Occidente, según la cual es cierto que la civilización occidental se halla enferma y sometida a una crisis, pero no de necesidad mortal, el capitalismo es una de las configuraciones de Occidente, el capitalismo devendrá en socialismo. Son las instituciones provenientes de Occidente las que están en crisis. Esto sucede porque Europa a través de la colonización expandió sus instituciones a todo el mundo; la crisis es europea en su origen, pero en verdad es una crisis mundial. Para Mariátegui la crisis era de índole económica. Pero a la vez política: la guerra y la situación posbélica habían creado una coyuntura explosiva y destruido las instituciones y finalmente la crisis se había expandido al terreno de las ideas: ella ha minado todas las antiguas certezas, dando lugar al escepticismo y relativismo y erosionando el racionalismo, el historicismo y positivismo precedentes. La crisis mundial es por tanto una crisis económica, política e ideológica.

1 Mariátegui, J. Carlos. (1994) 7 ensayos de la interpretación de la realidad peruana. Lima: Biblioteca Amauta. p. 231. 
Observaciones: Una vez más abundan las descripciones, pero faltan reflexiones críticas y propuestas sobre la real o supuesta crisis del capitalismo y la superación por el socialismo. Sobre las corrientes de pensamiento contemporáneo como el espiritualismo, pragmatismo, psicoanálisis, voluntarismo, etcétera, en el Amauta hay una asimilación crítica e interesante en el libro «Defensa del marxismo».

\section{Tercer artículo: Amauta como revista política y su visión de los problemas políticos internacionales}

Sobrevilla realiza un análisis político de la revista, a diferencia de los otros estudios de carácter más literario. Dice que Amauta formaba parte del proyecto político de José Carlos Mariátegui después de su regreso de Europa; que hacia 1925 la revista debía llamarse "Vanguardia» debido a su doble intención de expresar a las vanguardias artísticas y políticas, pero que finalmente Mariátegui se decidió por el título "Amauta» para vincular claramente el internacionalismo vanguardista con las raíces peruanas. La revista Amauta atravesó tres etapas: $1^{\mathrm{a}}$ de "definición ideológica», del primer número hasta el 16 (setiembre 1926-julio 1928), 2a "Revista Socialista», del número 17 al 29 (setiembre 1928-marzo 1930) y $3^{\mathrm{a}}$ "Revista de Clase», del número 30 al 32 (abril-mayo 1930, agosto-setiembre 1930).

Observaciones: Nadie cuestiona el hecho de que la revista Amauta formara parte del proyecto político de Mariátegui, por más que sea de carácter «literario». Por otra parte, Sobrevilla no menciona los dos tipos de prensa de los que hablaba Mariátegui: prensa de información y prensa de doctrina ${ }^{2}$. La primera es más comercial, "neutral», mistificadora de los hechos en algunos casos y la más eficaz propagandista de ideas conservadoras. La segunda es menos comercial, parcial, relativamente objetiva y propagandista de ideas progresistas. La revista Amauta y el periódico Labor que publicó Mariátegui en 1926 y 1928 respectivamente, fueron considerados por él como prensa más próxima a la doctrina que a la información, aunque Amauta parecía más académica, erudita, de ilustración integral y no dirigida exclusivamente a los «trabajadores manuales».

\section{Cuarto artículo: la tesis de Mariátegui sobre el carácter feudal de la economía colonial en el Perú}

A la pregunta: ¿fue la economía colonial peruana feudal? José Carlos dio una respuesta positiva en su esquema de la evolución económica del Perú. La palabra feudalismo es polisémica: podemos tomarla en un sentido histórico, como

2 Mariátegui, J. Carlos. (1979) Ideología y política. Lima: Biblioteca Amauta.pp. 174-178. 
refiriéndose a un fenómeno que se dio originalmente en Europa o en un sentido marxista como designando un modo de producción determinado. Sobrevilla opina que no se puede seguir sosteniendo la tesis de Mariátegui sobre el carácter feudal del Perú colonial ni en sentido histórico ni en sentido marxista ya que: 1) la caracterización del feudalismo europeo no se cumple en el caso del llamado por Mariátegui feudalismo colonial peruano, y 2) los rasgos del modo de producción feudal tampoco corresponden a la realidad histórica de la economía peruana. En resumen, para Sobrevilla la caracterización de Mariátegui es simplista porque no advierte la importancia de la actividad minera colonial, del comercio interprovincial, de la actividad de las haciendas y de los obrajes.

Observaciones: efectivamente, Mariátegui sostiene la tesis de que sobre los residuos de una "economía socialista», refiriéndose a la incaica, surgió una economía feudal que se ha prolongado hasta la República, en la que coexisten elementos de tres economías diferentes: en la sierra subsisten residuos vivos de economía comunista indígena, en la costa crece una economía burguesa sobre una economía feudal. Para justificar su tesis el Amauta aduce dos razones de orden económico: 1) la concentración de la tierra en unos cuantos propietarios, que conformaran la clase terrateniente o aristocracia feudal, cuyo propósito principal es vivir de las rentas y no desarrollar la producción o industria, 2) la servidumbre indígena a través del yanaconaje, es decir el sistema de explotación, llámese encomienda, corregimiento o reducciones, era con fines agrícolas y mercantiles. En conclusión, para Mariátegui en la etapa de la dominación colonial del Perú convergen elementos del sistema feudal, mercantilista y esclavista. Sin embargo en el análisis y exposición de su tesis es muy esquemático, falta argumentación y notamos ese afán por encasillar la economía peruana según los modos de producción europeos concebido por Marx. En ese sentido con este artículo, Sobrevilla, señala acertadamente las limitaciones conceptuales y argumentativas de Mariátegui.

\section{Quinto artículo: La visión del mito en José Carlos Mariátegui, Mariano Iberico y Luis Alberto Sánchez}

La reflexión sobre el mito en Mariátegui es una consecuencia de la lectura marxista del libro de Oswaldo Spengler "La decadencia de Occidente». El tema estricto del estudio de «La decadencia de Occidente» es el proceso final de la civilización occidental. El relativismo, el escepticismo, el subjetivismo serían los síntomas de esta situación. Según Mariátegui es cierto que la civilización occidental se halla enferma y sometida a una crisis, pero no de necesidad mortal. En verdad, es solo el capitalismo el que está en crisis. El capitalismo habrá de ser seguido por el socialismo. Para Mariátegui el mito es una concepción metafísica de la existencia que 
procura sentido a la vida. El gran mito de la época contemporánea -pensaba José Carlos- era el mito de la revolución social como toma del poder.

Para Mariano Ibérico el mito es un de las formas en que actúa creadoramente el espíritu, el mito posee dos condiciones: 1) la existencia de un alma popular, y 2) un pasado pre-temporal. Entonces, la existencia del mito revela la existencia de un pueblo, su ausencia significará que el pueblo no exista. Este sería el caso de Nuestra América ya que al no haber mito podemos colegir que no ha habido un pueblo y con ello un alma popular. Por último, en contra de lo afirmado por Ibérico, para Alberto Sánchez el mito existió en América Latina. Mitos fueron los de Manco Capac, Huiracocha, Pachacamac. Los mitos americanos son como los orientales, mitos donde la individualidad no posee importancia, por lo que no sabemos quiénes son sus autores. Sánchez encuentra que nos ha faltado una clase culta. Su ausencia explicaría que no hayamos tenido un gran mito al que el alma popular americana hubiera podido prestarte su adhesión incondicional.

Observaciones: Sobrevilla no justifica adecuadamente o trata de forzar la idea de que Mariátegui reflexiona sobre el mito como consecuencia de la lectura en clave marxista de "La decadencia de Occidente» de Oswaldo Spengler. Por el contario, es el mismo Mariátegui quien afirma haber reflexionado sobre el mito ${ }^{3}$ a raíz de la lectura de «Reflexiones sobre la violencia» de Georges Sorel, donde el pensador francés habla explícitamente del mito de la huelga general como mito revolucionario. Si no, veamos lo que dice: «Se puede hablar indefinidamente de revueltas sin provocar jamás un movimiento revolucionario, mientras tanto no haya mitos aceptados por las masas. Esto es lo que le otorga una importancia tan grande a la huelga general y lo que la convierte tan odiosa a los socialistas que tienen miedo de una revolución... Los mitos revolucionarios no son descripciones de cosa, sino expresión de voluntades. La utopía, por el contrario, es producto de un trabajo intelectual $»^{4}$ Por otra parte, para Iberico y Sánchez, el mito tiene un carácter popular colectivo e histórico y es la condición necesaria y suficiente para la existencia y trascendencia de un pueblo. En Mariátegui el mito también tiene un carácter popular, multitudinario e histórico, pero difiere en su aspecto revolucionario, ya que se trata de instaurar un orden social nuevo a través de una lógica y métodos violentos.

\section{Sexto artículo: González Prada y Mariátegui}

La semejanza entre los planteamientos de Mariátegui y González Prada son los siguientes: su visión del Perú como un país indígena y el tipo de solución

3 Mariátegui, J. Carlos. (1972) Alma Matinal. Lima: Biblioteca Amauta.pp28.

4 Sorel, George. (1985) Reflexiones sobre la violencia. Buenos Aires: La pléyade. p38. 
que ofrece al indio es básicamente económico y social. Por su parte Mariátegui señalaba que el problema primario del Perú era el problema indígena al que subyacía una causa económica: el problema de la tierra, por lo que la solución tenía que ser la devolución de las tierras de las que habían sido despojadas las comunidades indígenas. Una segunda semejanza es la crítica a la propiedad individual. González Prada opinaba siguiendo a Proudhon que la propiedad es un robo. De su parte, Mariátegui recordaba que en el Perú precolombino solo había existido la propiedad colectiva; señalaba que la propiedad recién había sido introducida en nuestro país por la conquista. Una tercera semejanza es la crítica de González Prada a la explotación del obrero por el capital: le parecía una continuación del vasallaje feudal. En los últimos tiempos, el ensayo de Mariátegui sobre la literatura ha sido muy criticado. Luis Loayza le ha objetado no estudiar la literatura como literatura sino como un documento político y social. Por su parte, Marcel Velásquez Castro sostiene que Mariátegui despreciaba la literatura oral, desconocía muchos textos significativos del período colonial, tenía prejuicios racistas contra los negros y los chinos, sobrevaloraba a algunos autores solo porque realizaban en sus obras consignas socialistas.

Observaciones: las críticas de Luis Loayza y Marcel Velásquez Castro no son del todo válidas por las siguientes razones: 1) para Mariátegui sería ingenuo pensar que los intelectuales son imparciales, carentes de convicciones religiosas y políticas, así estos no lo manifiesten explícitamente en sus obras, pues las obras literarias son un producto tanto individual (creaciones del autor) como histórico (ubicación espacial y temporal del autor ), y el hombre que las crea, las crea por y con algún interés, sea estético, epistémico, religioso, económico, social o político, pero siempre hay un interés de por medio. Por eso las obras literarias son documentos históricos que nos revelan una dimensión objetiva (contexto social y político) y subjetiva (biografía y psicología) del autor. Lo que Mariátegui hace en «El proceso de la literatura» es desentrańar los intereses subrepticios, ocultos y subyacentes en la literatura peruana que legitiman un estado de cosas, una clase social determinada, un régimen político o una tendencia ideológica. 2) la honestidad de Mariátegui es admirable ya que él mismo señala los criterios con los que analiza las obras y autores, a saber: unificar las concepciones estéticas con las concepciones morales, políticas, religiosas y hasta filosóficas que, sin dejar de ser concepciones estrictamente estéticas no pueden operar independientemente. Pero ¿̨por qué dice Mariátegui que su criterio es literario? Porque al no utilizar el esquema marxista de literatura feudal, burguesa y proletaria sus planteamientos no son calco y copia sino reformulación crítica, y porque él ya se esperaba la avalancha de críticas de los académicos universitarios, aunque esto no es tan relevante. Por consiguiente, 
en él opera algo que denomina, unimismamiento5. 3) afirmar que Mariátegui es racista es del todo malintencionado e imprudente ya que la única fuente de estas afirmaciones son unos pequeños párrafos en el que aparentemente hay un desprecio por el negro y el chino, por causa de su origen étnico, que a continuación transcribiré: «El aporte del negro, venido como esclavo, casi como mercadería, aparece más nulo y negativo aún. El negro trajo su sensualidad, su superstición, su primitivismo. No estaba en condiciones de contribuir a la creación de una cultura, sino más bien de estorbarla con el crudo y viviente influjo de su barbarie» ${ }^{6}$. Para alguien que desconoce de literatura marxista y que solo ha leído tendenciosamente «El proceso de la literatura» obviamente Mariátegui sería racista; no olvidemos que en «Ideología y Política» el Amauta escribe (la primera parte, pp. 21-46) el artículo titulado "El problema de la razas en América Latina», donde sostiene que el problema de las razas se resuelve liquidando la feudalidad ya que el indígena, el negro, el chino tienen en común la explotación en condiciones de servidumbre; no obstante, la principal fuerza productiva la proporciona el indígena. Asimismo, el prejuicio de la inferioridad racial es una invención de los intelectuales burgueses para encubrir y justificar el régimen feudal de la gran propiedad. Ahora bien, desde la perspectiva marxista que asume Mariátegui y tomando en cuenta que su propósito es generar las condiciones para una revolución democrática burguesa ${ }^{7}$ (primera fase) y proletaria (segunda fase), hay dos conceptos claves, el de cantidad y calidad en la lucha de clases, el de cantidad se refiere a quienes conforman la mayoría de explotados en el Perú, es decir, los indígenas, y el de calidad se refiere al grado de conciencia social, política y de clase, este es, el proletariado. Es por eso que Mariátegui propone una alianza por y para la revolución entre el proletariado y el campesinado indígena dado que la burguesía no es capaz de cumplir con su misión histórica, barrer la feudalidad. De ahí que los negros y los chinos no jueguen un papel trascendental en el proceso revolucionario, por razones de cantidad y calidad, es decir, no constituyen el grueso de la población y no tienen la madurez ideológica y política para conducir la revolución. En conclusión el Amauta no sería racista, sino un pensador que por el contexto comparte con Pedro Zulen, Manuel González Prada y otros, un "discurso racialista» ${ }^{8}$ basado en lecturas y experiencias personales, que lo llevan a establecer diferencias psicológi-

5 Mariátegui, J. Carlos. (1994) 7 ensayos de la interpretación de la realidad peruana. Lima: Biblioteca Amauta.p231.

6 Mariátegui, J. Carlos. (1994) 7 ensayos de la interpretación de la realidad peruana. Lima: Biblioteca Amauta.p342.

7 Mariátegui, J. Carlos. (1979) Ideología y Política. Lima: Biblioteca Amauta.p158-162.

8 Rojas, Joel. (2013) La educación y el racialismo en el discurso critico de Pedro Zulen durante el debate en el Centro Universitario a inicios del siglo XX. Solar, Revista de Filosofía Iberoamericana No 7, Lima. 
cas e históricas, accidentales más no esenciales, entre indios, negros y chinos. Pero no olvidemos que la principal razón de su «desprecio» por el negro y el chino es económica (participación de la producción), social (aspecto demográfico: mayoría o minoría), política (grado de conciencia clasista y experiencia en la lucha), y no étnica (rasgos fenotípicos: color de piel, etcétera).

\section{Séptimo artículo: Mariátegui e Iberico}

El objetivo del texto es mostrar la colaboración intelectual, la influencia recíproca y muy fugaz entre Mariátegui e Iberico. Iberico se reunió desde 1925 con Alberto Ureta, Alberto Ulloa y Mariátegui debido a que eran miembros del Consejo de Redacción del "Mercurio Peruano», revista fundada en 1918 por Víctor Andrés Belaúnde, quien en julio de 1921 había sido exiliado del Perú por el régimen de Leguía. Por su parte Mariátegui publicó en su recién constituida casa «Editorial Minerva» el libro de Iberico «El nuevo absoluto» en enero de 1926. Pero además de la colaboración intelectual entre ambos, es bien sabido que el Amauta influyó políticamente sobre Iberico. Prueba de ello es que la revista adquiere una clara orientación de izquierda. Fue demasiado para Belaunde, quien por entonces tenía una posición de centro derecha y desautorizó la nueva línea adoptada por la revista. Finalmente, y luego de haber estado tan próximo a Mariátegui entre 1925 y 1929, Iberico no lo menciona nunca en sus textos posteriores, borrándose toda influencia de José Carlos sobre su obra. ¿Qué pudo ser lo que llevó a Mariátegui a publicarle a Ibérico El nuevo absoluto? Y ¿qué influencia pudo haber ejercido el filósofo sanmarquino sobre el Amauta? En opinión de Sobrevilla, Mariátegui lo hace porque este era hacia 1925/26 una figura intelectual joven, en plena producción y que por entonces gozaba de un gran prestigio en Lima y en el Perú en general. En cuanto a la segunda pregunta, Sobrevilla formula la hipótesis de que Iberico contribuyó a que Mariátegui cambiara su opinión sobre la importancia de la filosofía bergsoniana para la historia de su época, y a que leyera al pensador francés.

Observaciones: la conjetura de Sobrevilla sobre el cambio de opinión y valoración de la filosofía bergsoniana en Mariátegui, provocada por la influencia de Iberico no es satisfactoria porque simple y llanamente no es del todo cierta. El prurito o curiosidad intelectual por leer a Bergson se lo contagió probablemente Iberico, pero el vivo interés por asimilar críticamente la filosofía de Bergson dentro del marxismo, procede de la lectura de "Reflexiones sobre la Violencia» de George Sorel, donde el autor presenta a Bergson como el intelectual que nos ha enseñado que la religión no es lo único que ocupa un lugar en la conciencia profunda, 
sino que también los mitos revolucionarios tienen el mismo título que aquella?. Prueba de ello, es la mención que hace Mariátegui de Sorel en su artículo Henri de Man y la «crisis» del marxismo de 1926, año en que Mariátegui publica en su Editorial Minerva «El nuevo absoluto» de Iberico. En conclusión, el contacto y posterior asimilación crítica de Mariátegui con la filosofía de Bergson procede de dos canales: Iberico o Sorel. Sostengo que es por medio de Sorel porque Mariátegui lo reconoce como un "revisionista verdadero» ${ }^{10}$ en el sentido de renovación y continuación de la obra de Marx.

\section{Octavo artículo: La recepción de los 7 ensayos en las ciencias sociales perua- nas}

El texto se divide en cinco secciones, la primera se titula «Del descubrimiento de las ideas de Mariátegui a su redescubrimiento». Sobrevilla comienza por narrar los acontecimientos en torno a la muerte de Mariátegui, como el cambio de nombre del Partido Socialista en Partido Comunista, el cambio de orientación ideológica y política, así como también las críticas de Mirochesvki contra Mariátegui, que calificaba su posición de "populista». No solo se había expropiado el partido de Mariátegui sino también su propio pensamiento asignándole uno que no se encuentra en sus escritos. Esta operación resultó facilitada porque a su muerte José Carlos solo había publicado dos libros: «La escena contemporánea» (1925) y «7 ensayos de interpretación de la realidad peruana» (1928) y una multitud de ensayos desperdigados en periódicos y revistas. Todo lo anterior explica que, como escribía Alberto Flores Galindo, Mariátegui era un desconocido en el Perú de 1940. La situación cambió radicalmente cuando, a partir de 1943, la familia Mariátegui decide reeditar esta vez en forma completa los 7 ensayos.

La segunda sección se titula Los periodos de la recepción de los 7 ensayos. Sobrevilla distingue tres periodos: A) La recepción inmediata de los 7 ensayos, luego de su publicación a inicios de octubre de 1928. La publicación produjo diversas reacciones; las más importantes fueron las siguientes: a) La reacción conservadora representada por la respuesta del intelectual católico Víctor Andrés Belaunde en su libro La realidad Nacional. b) La reacción aprista, movimiento que inicialmente trató en lo político de establecer distancias con Mariátegui y luego se esforzó por recuperar su figura para el aprismo a partir de la década del 50. c) La reacción del marxismo ortodoxo que pasó a secuestrar el pensamiento de Mariátegui. B) El segundo periodo fue posible gracias a la republicación del libro en forma completa por la familia de Mariátegui en 1943. Podemos fijar el término

9 Sorel, George. (1985) Reflexiones sobre la violencia. Buenos Aires: La pléyade. p40.

10 Mariátegui, J. Carlos. (1967) Defensa del marxismo. Lima: Biblioteca Amauta.p16. 
inicial de este periodo hacia finales de los años 50. El primer trabajo con cierta amplitud consagrado al Amauta fue el de Carlos Núñez Anavitarte «Mariátegui y el descentralismo». C) el tercer periodo se inicia hacia el año 1989 con la crisis del marxismo realmente existente. Manifiesta Sobrevilla que esta crisis ofrece la oportunidad de reconstruir el genuino pensamiento de Mariátegui más allá de las presiones por confirmarlo como un marxista ortodoxo o heterodoxo.

La tercera sección se titula «El esquema de la evolución económica». Sobrevilla sostiene: primero, que no puede asignarse al Imperio incaico el carácter de una economía comunista. Segundo, el día de hoy con la cantidad de datos nuevos de que se dispone sobre la colonia peruana se ha vuelto insostenible afirmar que tuvo un carácter feudal. Tercero, en caso de que esto fuera así, mal puede afirmarse que la economía peruana republicana siguió siendo feudal. La cuarta sección se titula: Regionalismo y centralismo». Para Mariátegui la polémica entre federalismo y centralismo era anacrónica. El federalismo favorecía al gamonalismo o sea a los grandes terratenientes y el centralismo buscaba una íntegra revisión política económica de la realidad del Perú, siendo sus bases las regiones y no los «departamentos» que se derivan de las antiguas intendencias. La quinta y última sección se titula: «El proceso de la literatura». En esta sección Sobrevilla se limita a describir las críticas que se han formulado hasta los tiempos actuales. Como por ejemplo, según Tamayo Vargas, dicho ensayo está sujeto a «multitud de contradicciones y superaciones en el campo de la crítica». Asimismo, Estuardo Núnez sostenía que dicho texto mariateguiano era insuficiente y arbitrario. Por otro lado, Antonio Cornejo Polar y Tomas Escajadillo han reivindicado los planteamientos de Mariátegui. Cornejo sostiene que la cuestión nacional de la literatura deja de ser en Mariátegui tema académico para adquirir contenido político y que a partir de ahí hay que entender la reivindicación de la literatura indigenista como cancelatoria del periodo colonial y como superadora del cosmopolita. 